\title{
Bruising of erect penis: a rare complication of advising young, adult, male, cervical spinal cord injury patient to lie prone for relief of pressure on sacral region
}

Spinal Cord (2003) 41, 653-654. doi:10.1038/sj.sc.3101531

When patients with spinal cord injury develop a pressure mark or pressure sore over the sacral region, they are advised to lie on their stomach or on their sides so that the sacral region is relieved of pressure. Health professionals are often conscious of the cardiac and respiratory complications of prone position and take suitable precautions to avert mishaps in the patients with cervical spinal cord injury who are advised to lie on the stomach. We were caught unawares when a young, adult, male patient with cervical spinal cord injury, who was lying prone in order to relieve pressure over the sacral region, developed a complication related to positioning on the bed.

A 25-year-old male sustained C-6 tetraplegia (ASIA B) after a diving accident 4 years ago. He managed his bladder with suprapubic cystostomy. He would get erection of penis spontaneously. He developed pain over the sacral region on the right side and noticed a pressure mark. Therefore, he was lying prone on a pressurerelieving mattress with the penis pointing downwards towards his feet. He developed erection of penis. The penis was pushed against the bed. Subsequently he fell asleep. In the morning, he noticed bruising of the dorsal surface of the penis over a large area (Figure 1). There was no angular deformity of penis. There was no bleeding per urethra.

This patient was not taking anticoagulants or aspirin. He had no history of bleeding diathesis. There was no bruising or petechial haemorrhages in any other part of the body. There was no family history of bleeding tendency.

Ultrasound scan of penis was performed to exclude injury to corporal bodies. Luckily, there was no fracture of corpora cavernosa (Figure 2). Full blood count as well as coagulation profile was normal. Bruising over the dorsal surface of penis subsided gradually over a period of 2 weeks and did not require any active treatment.

Health professionals caring for cervical spinal cord injury patients must be aware that some patients may develop spontaneous erection of penis. This case illustrates a rare complication of spontaneous erection of penis in a cervical spinal cord injury patient, who was lying prone for relief of pressure over sacrum. Before advising a young, male, adult, patient with tetraplegia to lie prone, the health professional should consider the following aspects:

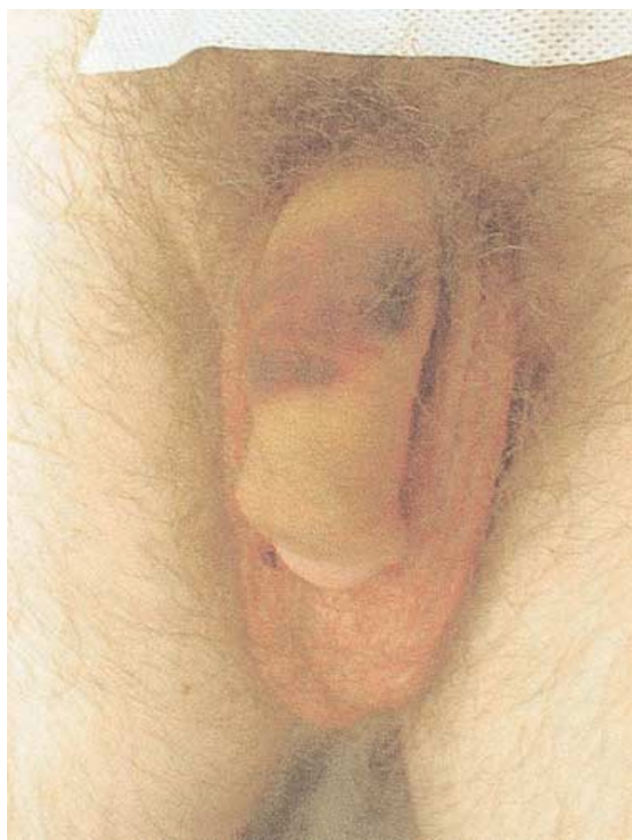

Figure 1 Clinical photograph of penis: there is extensive bruising of penis

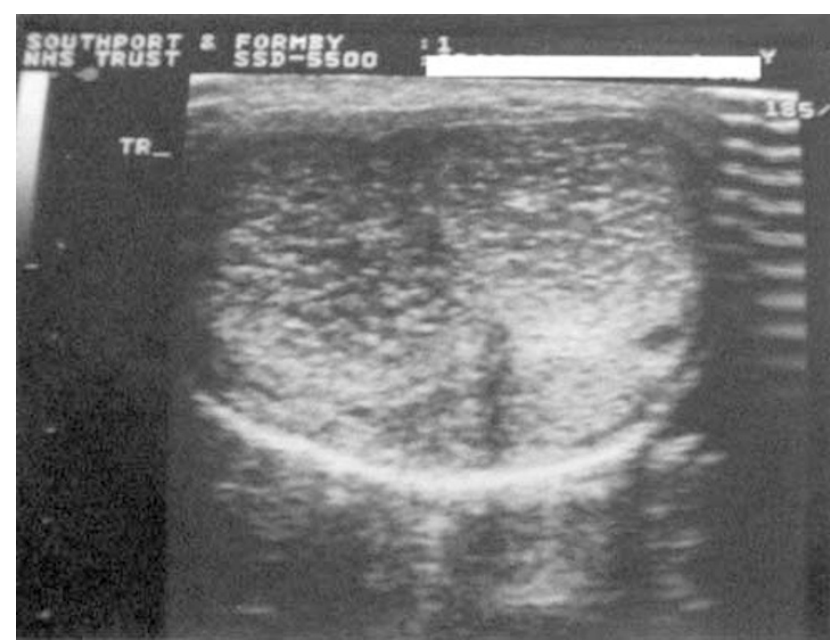

Figure 2 Ultrasound scan of penis: the corpora cavernosa appear normal 
- Does this patient get spontaneous erection of penis?

- If the patient is likely to develop penile erections, foam wedge(s) may be kept under the suprapubic region or the iliac crest in order to avoid pressure of torso upon penis. This will ensure that the penis is not forcibly bent should the patient get erection of penis while lying on his stomach.

- The patient may be advised to lie in a tilted or sloping manner so that the penis is not forcibly bent by the weight of the body if tumescence occurs.

- When a young, male, adult, tetraplegic patient is advised to lie prone, health professionals should remind the patient and the carers to check the position of penis. This is comparable to the maxim that the carers make sure that the scrotum is not trapped when a patient with high cervical spinal cord injury is sat up on the chair. ${ }^{1}$ Should a patient get erection of penis while lying prone and the erect penis gets forcibly bent by the weight of the body, the corpora may be fractured or, there may be extensive bruising of penis as indeed happened to this patient.

S Vaidyanathan, BM Soni, PL Hughes, J Bingley, JWH Watt and G Singh Regional Spinal Injuries Centre District General Hospital, Town Lane, Southport Merseyside PR8 6PN, UK

\section{References}

1 Vaidyanathan S et al. Blunt trauma to scrotum in men with spinal cord injury after they had completed rehabilitation in a spinal unit. Spinal Cord 2001; 39: 442-448. 